\title{
Basic physical properties of the close binary V497 Cep in the open cluster NGC $7160^{\star}$
}

\author{
K. Yakut ${ }^{1}$, A. E. Tarasov ${ }^{2,3}$, C. İbanoğlu ${ }^{1}$, P. Harmanec ${ }^{4,5}$, B. Kalomeni ${ }^{1}$, D. E. Holmgren ${ }^{6,7}$, \\ H. Božici ${ }^{8}$, and P. Eenens ${ }^{9}$
}

\author{
1 University of Ege, Faculty of Science, Department of Astronomy and Space Sciences, 35100 Bornova, İzmir, Turkey \\ 2 Crimean Astrophysical Observatory, 98409 Nauchny, Crimea, Ukraine \\ 3 Isaac Newton Institute of Chile, Crimean Branch, 98409 Nauchny, Crimea, Ukraine \\ 4 Astronomical Institute of the Charles University, V Holešovičkách 2, 18000 Praha 8, Czech Republic \\ 5 Astronomical Institute of the Academy of Sciences, 25165 Ondřejov, Czech Republic \\ ${ }^{6}$ SMART Technologies, Inc., Bay 8, 1460 - 28 Street NE, Calgary, Alberta, T2A 7W6, Canada \\ 7 Dominion Astrophysical Observatory, Canada \\ ${ }^{8}$ Hvar Observatory, Faculty of Geodesy, Zagreb University, Kačićeva 26, 10000 Zagreb, Croatia \\ 9 Departamento de Astronomia, Universidad de Guanajuato, Apartado 144, 36000 Guanajuato, GTO, Mexico
}

Received 28 March 2003 / Accepted 6 May 2003

\begin{abstract}
New light and radial-velocity curves of V497 Cep, a binary in the open cluster NGC 7160, were obtained and the linear ephemeris of the system was refined to HJD $($ Min I $)=(2446299.1596 \pm 0.0064)+(1.2028287 \pm 0.0000015) \times E$. The first light and radial-velocity curve solutions allowed us to derive the basic physical properties of this astrophysically important binary. It was found that the observed light variation of V497 Cep consists of a strong ellipticity effect and a small contribution from grazing eclipses. A comparison of masses and radii of V497 Cep with theoretical evolutionary tracks indicates that both binary components are very close to the zero-age main sequence. A comparison of disentangled line profiles of the He I 6678 line with synthetic, rotationally broadened line profiles indicates that the rotation of both stars is synchronized with the orbital revolution as expected. This finding increases the credibility of our solutions. We find $E(B-V)=0.39$. The distance to the cluster NGC 7160 was found to be about $760( \pm 100)$ pc which agrees well with other available estimates.
\end{abstract}

Key words. stars: binaries: eclipsing - stars: binaries: spectroscopic - stars: individual: V497 Cep -

Galaxy: open clusters and associations: individual: NGC 7160

\section{Introduction}

V497 Cep (BD+61 2213 , NGC $7160 * 4)$ is an early-type, short-period binary system which is a confirmed member of the open cluster NGC 7160. The binary is a reddened B3 star and the fourth brightest member of the cluster. Its magnitude and colours as well as the period of light variability were derived recently by Harmanec et al. (1999) as $V=8 \mathrm{~m} .961$, $B-V=+0^{\mathrm{m}} .191, U-B=-0^{\mathrm{m}} .466$, and $P=1.2028251$.

$\mathrm{BD}+61^{\circ} 2213$ was first reported to be a light variable by Hill (1967a,b). He tentatively classified it as an eclipsing variable, not on the basis of his photometry but on the basis of an earlier report of its radial-velocity (RV hereafter) variability by Hayford (1932). Hill et al. (1976) published individual

Send offprint requests to: $\mathrm{K}$. Yakut, e-mail: yakut@astronomy.sci.ege.edu.tr

* Tables 1 and 2 are only available in electronic form at the CDS via anonymous ftp to cdsarc.u-strasbg.fr $(130.79 .128 .5)$ or via http://cdsweb.u-strasbg.fr/cgi-bin/qcat? J/A+A/405/1087 photometric observations of the star, again indicating mild light changes.

Overlooking these reports, Harmanec et al. (1994) used the star occasionally as one of the $U B V$ standards in the course of their systematic observations of Be and Ap stars and some binary systems at Hvar and Skalnaté Pleso observatories. Alerted by larger systematic deviations of this "standard" during the $1998 U B V$ observations, Harmanec et al. (1999) homogenized and re-analyzed all available photometric observations, including a rich set of 107 good Hipparcos observations, and concluded that the star varies periodically, with a period of $1.2028251^{1}$. Harmanec et al. suggested that the star is more probably an ellipsoidal variable than an eclipsing binary. They appealed to spectroscopists and photometrists to collect new accurate observations which would allow to improve the knowledge of the basic physical elements of the binary and, consequently, also of the distance to cluster NGC 7160.

1 At about the same time, variability was also independently recognized by the Ukrainian observers. 
Subsequently, Kazarovets et al. (2000) assigned the object a variable-star name V497 Cep.

This prompted us to include V497 Cep into our spectroscopic and photometric observing programs at several observatories. Preliminary results of an analysis of spectral observations secured at Crimean Astrophysical Observatory and Dominion Astrophysical Observatory (DAO hereafter) were published in a poster paper by Holmgren \& Tarasov (2000). They arrived at RV semiamplitudes of 165 and $228 \mathrm{~km} \mathrm{~s}^{-1}$, for the primary and secondary, respectively.

The aim of this paper is to present and analyze new light and RV curves of the system and to derive for the first time also the absolute dimensions of the system.

\section{Observations}

\subsection{Photometric observations}

New systematic $B V$ observations of V497 Cep were carried out at Ege University Observatory. The 0.48-m Cassegrain reflector equipped with a SSP-5 photometer and Johnson $B$ and $V$ filters was used. The comparison star already used by Harmanec et al. (1999), HD 208440 = NGC 7160*3, was used. Its all-sky $U B V$ magnitudes derived by Harmanec et al. were adopted. V497 Cep was observed on 10 nights between July 31, 1999 and October 17, 2000. All differential observations were corrected for atmospheric extinction, using the extinction coefficient derived for each night of observation from the observations of the comparison star. A total of 270 observations in $B$, and 290 in $V$ band were obtained.

Additionally, all $U B V$ observations homogenized and used by Harmanec et al. (1999), together with a few new observations secured at Hvar and San Pedro Mártir observatories since then, were also used. Altogether, we accumulated $492 \mathrm{~V}, 365 \mathrm{~B}$ and $95 \mathrm{U}$ observations, covering the period HJD 2441499.8-2451843.8. For convenience of future investigators, we publish all these observations in Table 1.

Initially, the phase of each observation was calculated using the light elements given by Harmanec et al. (1999):

$\operatorname{HJD}(\operatorname{Min} I)=2446299.237+1^{\mathrm{d}} .2028251 \times E$.

All new Ege University Observatory $V$ and $B$ observations of V497 Cep are plotted in Fig. 1 vs. phase of ephemeris (1).

\subsection{Spectroscopic observations}

New electronic spectra of V497 Cep were obtained using two instruments, the $2.6 \mathrm{~m}$ telescope and spectrograph of the Crimean Astrophysical Observatory, and the $1.2 \mathrm{~m}$ telescope and coude spectrograph of the Dominion Astrophysical Observatory.

Crimean observations were secured at two spectral regions: near $\mathrm{H} \alpha$ and near He I $6678 \AA$. Only three $\mathrm{H} \alpha$ spectra were obtained, in June and July 1997. In the He I $6678 \AA$ region, 13 spectra were accumulated: 9 of them in OctoberNovember 1997 and 4 in July 1998. The detector was a CDS 9000 CCD array with $1024 \times 256$ pixels. All the observations were obtained in the second order of a diffraction grating with a

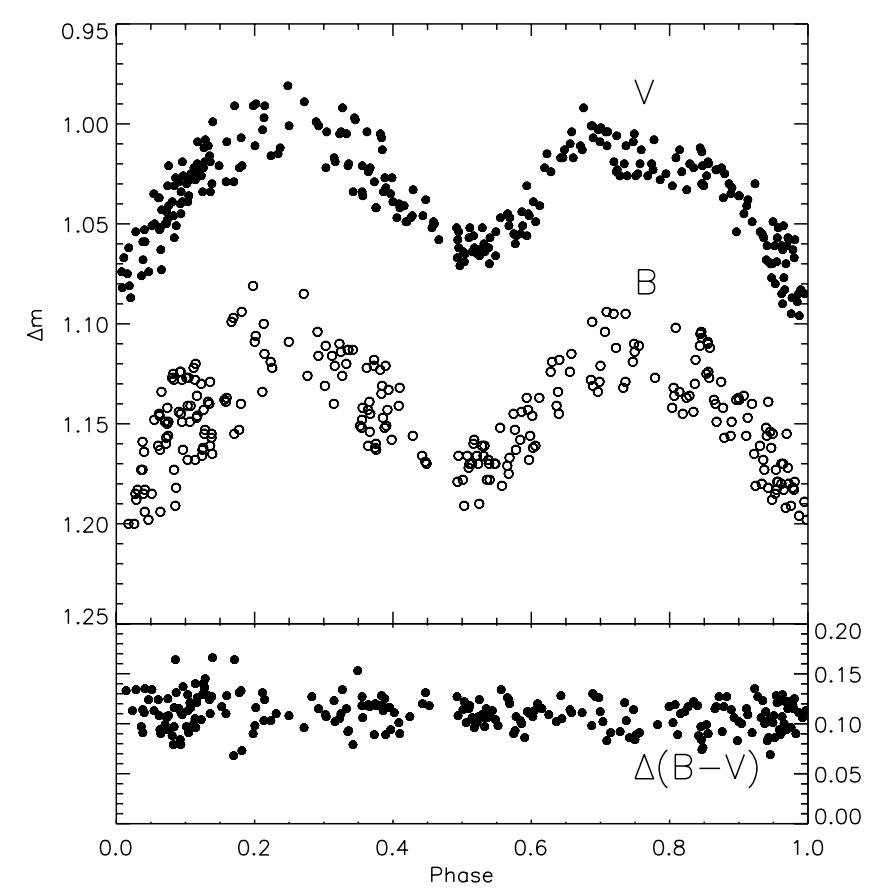

Fig. 1. The $B$ and $V$ light curves of V497 Cep, based on Ege University observations.

reciprocal dispersion of $3 \AA \mathrm{mm}^{-1}$. The spectral resolution was about 30000 and each spectrum covers about $60 \AA$. The exposure time ranged from 30 to $60 \mathrm{~min}$, depending on the weather conditions. All spectra were reduced using the standard techniques: dark-current subtraction and flat-field division. The subsequent reduction included the following procedures: removal of cosmic-ray spikes; sky background subtraction; normalization to the continuum approximated by a third-degree polynomial; and wavelength calibration using a ThAr comparison lamp, with an accuracy better than $1.5 \mathrm{~km} \mathrm{~s}^{-1}$. Wavelength scale and calculated Julian dates (JDs hereafter) were corrected to the barycenter of solar system.

First 17 DAO spectra were obtained in July 1999 using the 1.2-m telescope, $3261 \mathrm{H}$ spectrograph, and the UBC $4096 \mathrm{CCD}$ with $4200 \times 20$ pixels. The wavelength region was centred on $\mathrm{H} \alpha$, and each spectrum covered approximately $1200 \AA$. The reciprocal dispersion was approximately $20 \AA \mathrm{mm}^{-1}$. An exposure time of about $1800 \mathrm{~s}$ was used. Four additional spectra were secured in the year 2000. The data were reduced in the usual fashion using the MIDAS package. The bias and flat-field frames were averaged and used to remove the bias level and to remove the detector response from all stellar exposures. Each stellar frame was also cleaned of cosmic-ray spikes. One-dimensional spectra were then extracted and wavelengthcalibrated. Finally, all stellar spectra were rectified using a spline interpolation. All wavelength scales were adjusted for heliocentric radial velocity.

\section{Radial velocity analysis}

Already the first inspection of the absorption lines of $\mathrm{H} \alpha$ and He I 6678 revealed the presence of line doubling outside the 


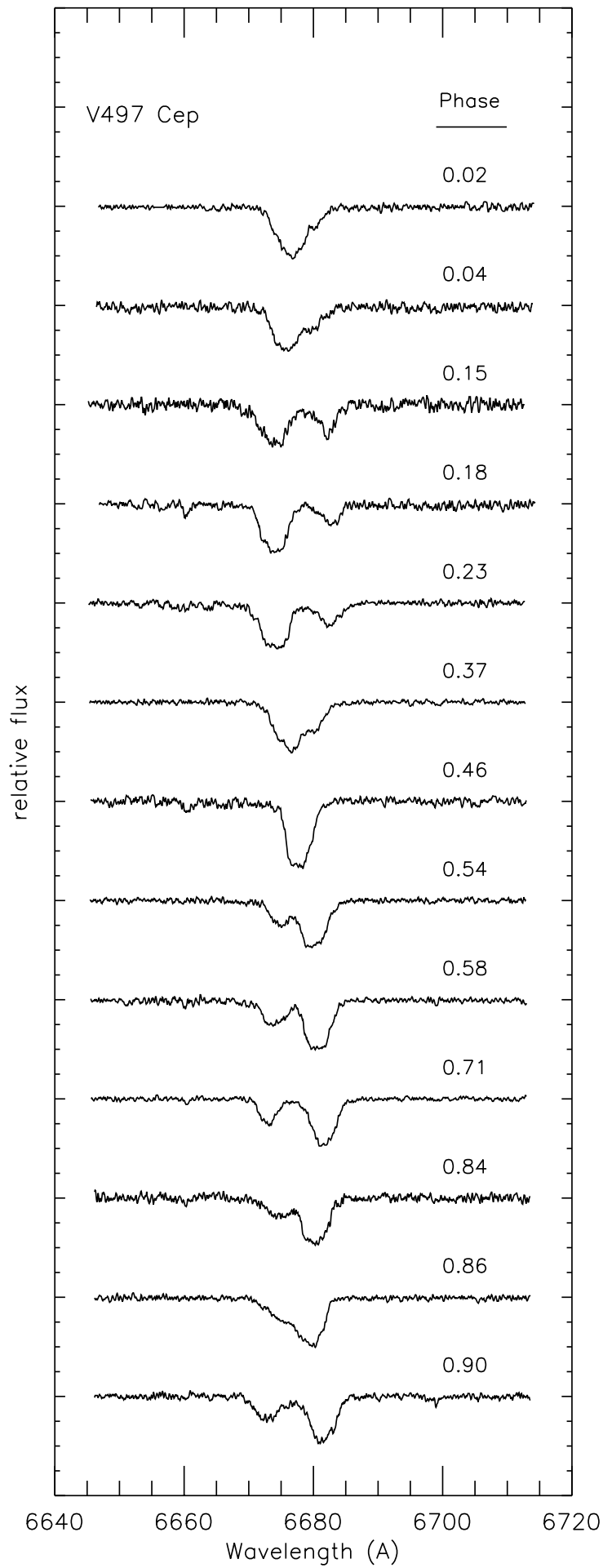

Fig. 2. Selected He I 6678 profiles of V497 Cep. The profiles are identified by phase according to ephemeris (1). Longer tick marks on the flux scale are separated for 0.2 of relative flux units.

eclipses, indicative of a double-lined binary with similar spectral types (cf. Figs. 2 and 3).

In all analyses, the Crimean spectra were given 5 times higher weight than the DAO spectra to take their much better linear dispersion into account. All spectra were imported into reduction program SPEFO (Horn et al. 1996) and the radial velocities were determined from the comparison of direct

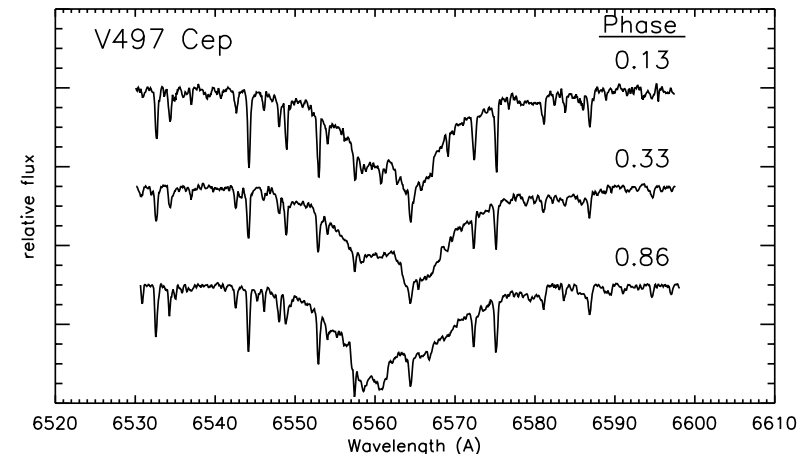

Fig. 3. Selected $\mathrm{H} \alpha 6563$ profiles of V497 Cep. The profiles are identified by phase according to ephemeris (1). Longer tick marks on the flux scale are separated for 0.2 of relative flux units.

and mirror image of each line profile. Heavily blended profiles near conjunctions were not measured. Following Horn et al. (1996) we also measured a selection of reliable telluric lines for all DAO and Crimean $\mathrm{H} \alpha$ spectra and used the differences of the mean telluric velocity and the calculated heliocentric correction to bring the data onto a joint RV zero point. Applying these corrections, we created input data for KOREL disentangling program (Hadrava 1995, 1997) and derived orbital solution and disentangled line profiles for the He 6678 line which is more suitable for the mass determination than the intrinsically broader $\mathrm{H} \alpha$ line. For comparison, we also used program FOTEL (Hadrava 1990) for the orbital solution based on classical RV measurements.

Individual radial velocities derived by direct measurements and by KOREL are given in detail in Table 2. Orbital elements based on a FOTEL solution using classical RV measurements and those derived via KOREL are summarized in Table 3 and the radial-velocity curves of the He I 6678 line derived via direct measurements and FOTEL solution, and via KOREL disentangling are compared in Fig. 4. Note that KOREL can use even blended line profiles which accounts for a higher number of data points in the bottom panel.

He I 6678 line profiles of both binary components, disentangled by KOREL, are shown in detail in Fig. 5 .

We give two FOTEL solutions in Table 3, allowing for a difference in the zero point of RV scale in the first case (the region near the He I 6678 line is free of telluric lines and no check on the zero point is therefore possible there). One can see that the classical and KOREL solutions agree within the limits of quoted errors. However, the KOREL solution gives a much smaller rms error and should be preferred for the determination of the masses of both binary components.

Radial velocities of the $\mathrm{H} \alpha$ line give a similar solution but with slightly reduced amplitudes which is a known effect. There are too few spectra as yet to obtain a complete removal of the telluric spectrum near $\mathrm{H} \alpha$ with KOREL. We therefore postpone the study of the $\mathrm{H} \alpha$ profiles for a future study.

\section{The new ephemeris}

Having at our disposal a longer series of light and RV observations than that available to Harmanec et al. (1999), we decided 

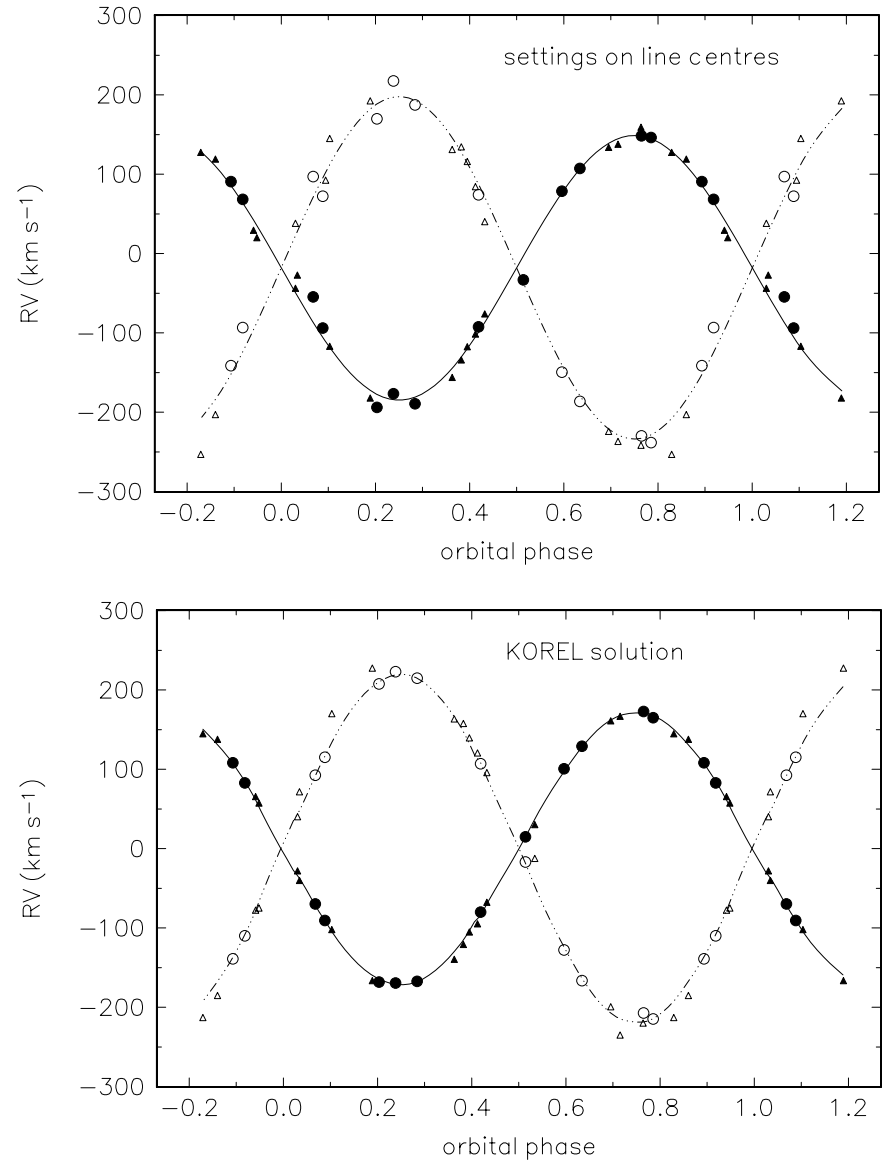

Fig. 4. A comparison of radial-velocity curves of the primary and secondary measured directly in SPEFO (upper panel) and based on KOREL disentangling (bottom panel). Filled symbols refer to the primary and the Crimean spectra are shown by circles, and the DAO spectra by triangles. The final ephemeris (2) was used.

to derive an improved ephemeris of the system. To this end, we used all $U B V$ observations together with all new RVs derived by KOREL for the He 6678 line and calculated a combined solution, using program FOTEL. Free convergence of the period, epoch, semiamplitudes of the RV curves, photometric radii and relative luminosities was allowed for and relative weight of photometry and radial velocities was chosen in such a way that each data set accounted for about one half of the sum of the squares of $\mathrm{O}-\mathrm{C}$ deviations.

We arrived at the following improved ephemeris

$$
\begin{aligned}
\operatorname{HJD}(\operatorname{Min} I)= & (2446299.1596 \pm 0.0064) \\
& +(1.2028287 \pm 0.0000015) \times E
\end{aligned}
$$

which we use in all subsequent analyses in this paper.

\section{Light-curve analysis}

Ten nights of Ege photometric $B$ and $V$-passband observations of V497 Cep, obtained in whole-night series, already shown in Fig. 1, are clearly the most suitable for the light-curve solution. We note that the orbital light curve in the $B$ passband indicates a great deal of scatter. The scatter of data at maximum light is about $0^{\mathrm{m}} .03$ in $V$ and $0^{\mathrm{m}} .05$ in $B$. In spite of this scatter, however,
Table 3. Radial-velocity solutions for the He I 6678 line, based on classical RV measurements (solutions 1 and 2) and on KOREL disentangling (solution 3). Period was kept fixed at 1.2028287 and zero eccentricity was assumed. All epochs are in HJD-2451022 and the quoted errors of the elements are rms errors. The rms error of the solution is the rms error of one point of unit weight.

\begin{tabular}{llll}
\hline \hline element & solution 1 & solution 2 & solution 3 \\
\hline$T_{\text {prim.min. }}$ & $0.6663 \pm 0.0038$ & $0.6662 \pm 0.0038$ & 0.6675 \\
\hline$K_{1}\left(\mathrm{~km} \mathrm{~s}^{-1}\right)$ & $166.7 \pm 5.3$ & $166.7 \pm 5.3$ & 171.7 \\
$K_{2}\left(\mathrm{~km} \mathrm{~s}^{-1}\right)$ & $215.9 \pm 8.4$ & $215.8 \pm 8.4$ & 219.6 \\
$\gamma_{\mathrm{CrAO}}\left(\mathrm{km} \mathrm{s}^{-1}\right)$ & $-17.2 \pm 3.1$ & - & - \\
$\gamma_{\mathrm{DAO}}\left(\mathrm{km} \mathrm{s}^{-1}\right)$ & $-20.6 \pm 2.8$ & - & - \\
$\gamma_{\text {joint }}\left(\mathrm{km} \mathrm{s}^{-1}\right)$ & - & $-17.9 \pm 2.6$ & $-19.2^{*}$ \\
No. of RVs & 55 & 55 & 60 \\
rms $\left(\mathrm{km} \mathrm{s}^{-1}\right)$ & 15.46 & 15.52 & 6.3 \\
\hline$M_{1} \sin ^{3} i\left(M_{\odot}\right)$ & 3.94 & 3.94 & 4.19 \\
$M_{2} \sin ^{3} i\left(M_{\odot}\right)$ & 3.04 & 3.04 & 3.28 \\
$A \sin i\left(R_{\odot}\right)$ & 9.09 & 9.09 & 9.30 \\
\hline
\end{tabular}

* Note that KOREL solution itself cannot give the systemic velocity. The value we quote is based on the mean of RV measurements of positions of KOREL-disentangled profiles of both binary components in SPEFO.

both the $V$ and $B$ observations clearly define a $\beta$ Lyr-type light curve with two minima and maxima. The depths of the primary and secondary minima are about $0^{\mathrm{m}} .09$ and $0^{\mathrm{m}} .07$ in $V$, and $0^{\mathrm{m}} .08$ and $0^{\mathrm{m}} .06$ in $B$, respectively. While the $B$ light curve has a symmetric shape, this is not so for the $V$ light curve: its maximum $I$ following the primary eclipse is higher than the other one. We note that such a variation with large scatter is characteristic of some known Be binaries. There is, however, no evidence of observable Balmer emission in the currently available spectra of V497 Cep. Yet, the observed scatter may perhaps indicate the presence of some circumstellar matter within the system. We, therefore, urge photometric observers in excellent observing sites to carry out further checks on the reality of this phenomenon.

The observed character of new light curves does not correspond to what would be expected for an ellipsoidal variable, advocated by Harmanec et al. (1999).

The solution of the new light curves were obtained using the latest version of the Wilson-Devinney code (Wilson \& Devinney 1971; Wilson 1994). For the main-sequence stars of spectral types B0 to A0, one can estimate the interstellar reddening, using the reddening-free quantity $Q=(U-$ $B)-0.72(B-V)$. According to the standard observed $U B V$ colours of V497 Cep obtained by Harmanec et al. (1999) we get $Q=-0.604$ which corresponds to a B3V star (Drilling $\&$ Landolt 2000). According to the mass of the primary component its effective temperature was assumed to be $19500 \mathrm{~K}$, following Drilling \& Landolt (2000), and it was kept fixed during the light-curve solution. The limb darkening coefficients of $x_{1}=0.441$ and $x_{2}=0.388$ (Claret 2000), albedos $A_{1}=A_{2}=$ 1.0 (Rucinski 1969) and gravity exponents of $g_{1}=g_{2}=1.00$ (von Zeipel 1924) were assumed and kept fixed. The brightness 


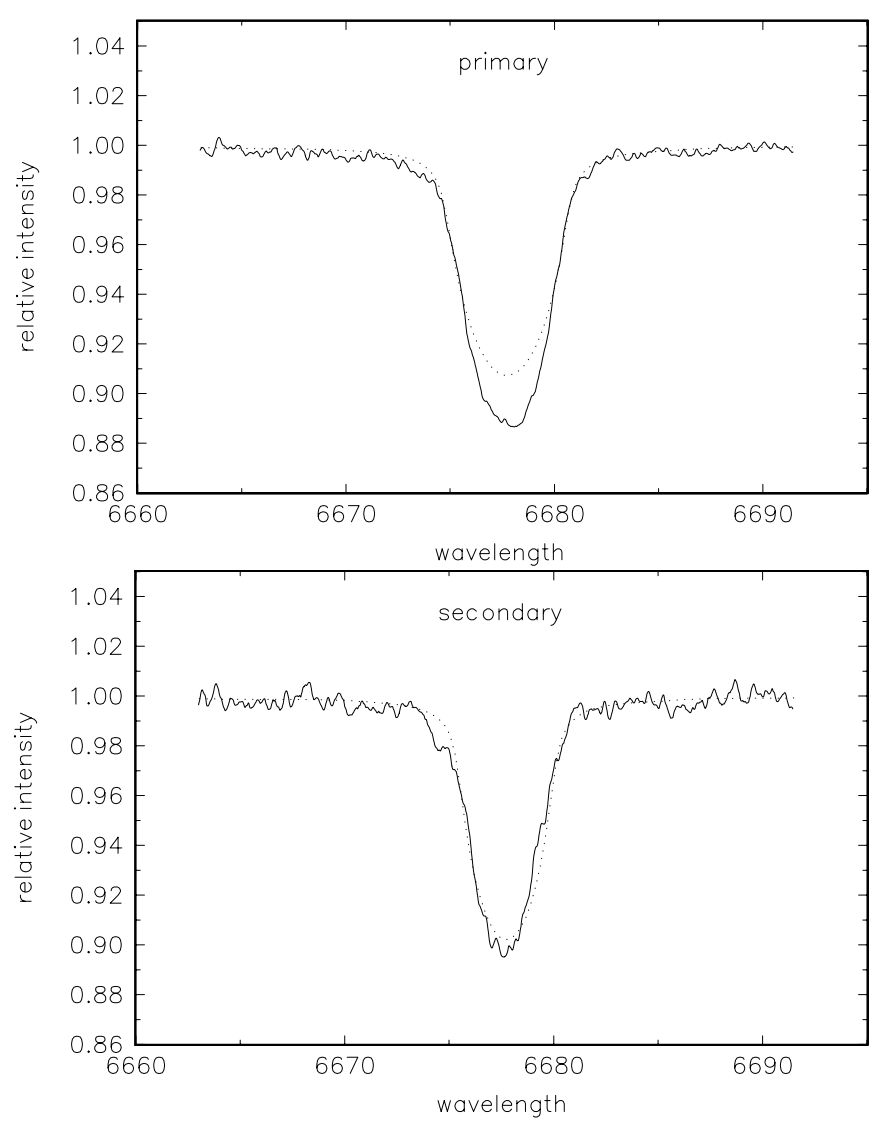

Fig. 5. He I 6678 line profiles of primary and secondary disentangled via KOREL (solid lines) are compared with synthetic LTE line profiles shifted for the systemic velocity of $-19 \mathrm{~km} \mathrm{~s}^{-1}$ and rotationally broadened to projected rotational velocities of 132 and $104 \mathrm{~km} \mathrm{~s}^{-1}$, for the primary and secondary, respectively, which correspond to spin-orbit synchronization. Wavelengths are in $\AA$ and the disentangled profiles from KOREL were normalized using the luminosity ratio from the $V$ light-curve solution - see the text for details.

of V497 Cep varies continuously in all orbital phases. A model light curve of a pure reflection effect has always an almost sinusoidal shape. Different choices of gravity- and limb-darkening coefficients led to only minute modifications. The deviations from a pure sinusoidal shape increase slightly with increasing orbital inclination in the sense that maximum become narrower. However, the observed light curve has a maximum which is almost equally broad as the minimum. Therefore, a satisfactory description of the light curve can only be achieved if one allows for some ellipticity, acting together with the reflection.

In order to match the observed light curve, the model requires a trade off between several parameters. First of all, the inclination of the orbit must be such to allow grazing eclipses to occur. The secondary component should have a similar radius and effective temperature as the primary, given the observed similarity in the depths of both eclipses. Having these constraints in mind, we used mode 2 of the Wilson-Devinney code (suitable for detached binaries with no constraints on surface potentials) to find the elements leading to the optimal agreement between model light curve and observations. A circular orbit and a black-body radiation were assumed for both stars.
The following elements were adjusted during the solution: orbital inclination $i$, temperature of the secondary $T_{2}$, dimensionless surface potentials $\Omega_{1}$ and $\Omega_{2}$, and the relative luminosities of the components in respective passbands, $L_{1}$ and $L_{2}$. The mass ratio of 0.783 is well determined by our RV solution 3 . It was, therefore, kept fixed in the light-curve solution. Since the $V$ light curve has unequal maxima, only the $B$ light-curve solution was used to obtain the geometrical and physical elements of the system. The functional dependencies of the effective temperature on mass and spectral type differ significantly from one calibration to another, especially for the massive stars. The results of the light curve analysis will of course depend on the adopted temperature of the primary component. Various effective temperatures for the primary component from 17000 to $23000 \mathrm{~K}$ were taken for the analysis of the light curve. The best fit, minimum squared residuals, was obtained at $19500 \mathrm{~K}$, which corresponds to a $\mathrm{B} 3 \mathrm{~V}$ star appropriate to the $Q$ value. The best-fit values obtained after several dozen of iterations with the DC code are listed in Table 4. The uncertainties of the parameters are formal errors which are probably unrealistically low. The WD code tends to give small formal errors of the derived parameters even for data with a larger scatter. The theoretical and observed $B$ light curves are compared in Fig. 6. We carried out, however, solutions of both, $B$ and $V$ light curves and obtained the fractional luminosities of the primary component as 0.654 and 0.651 in $B$ and $V$, respectively. In spite of all effort, we were unable to obtain a reliable solution for the limited number of more noisier $U$-passband observations and postpone that task until new, more accurate photoelectric observations will become available.

Our solution indicates that the orbital inclination is about $58^{\circ}$ and only grazing partial eclipses occur. Our model explains the observed light curve as being due to the combined effect of partial eclipses and ellipsoidal variations in a system consisting of two early B type components with similar temperatures.

\section{Basic physical properties of the system}

\subsection{Stellar masses and radii}

The masses of the primary and secondary stellar components can be determined because the inclination angle is already known from the light curve analysis. Assuming a circular orbit and combining the orbital elements obtained from the light curve analysis with those of RVs analysis one can find the stellar masses and radii in solar units. Relative side radii were used to derive the absolute radii. Basic macroscopic properties of both binary components are listed in Table 5. It should be stated here that the uncertainties of the absolute parameters are underestimated and mostly depended on the fractional parameters derived light curve analysis. In Fig. 7, the positions of binary components are compared with theoretical evolutionary tracks in the HR diagram and radius vs. effective temperature diagram based on Schaller's et al. (1992) evolutionary models for the main sequence stars. One can see that the masses of both binary components agree very satisfactorily with their positions in both diagrams. It is also clear that both components 
Table 4. The $B$ light-curve solution carried out with WD code.

\begin{tabular}{lll}
\hline \hline Parameter & Value & $\sigma$ \\
\hline$i\left(^{\circ}\right)$ & 57.9 & $\mp 0.3$ \\
$T_{2}(\mathrm{~K})$ & 17756 & $\mp 405$ \\
$\Omega_{1}$ & 3.825 & $\mp 0.106$ \\
$\Omega_{2}$ & 4.090 & $\mp 0.206$ \\
$\left(\frac{L_{1}}{L_{1}+L_{2}}\right)$ & 0.654 & $\mp 0.014$ \\
$r_{1}($ pole $)$ & 0.325 & $\mp 0.002$ \\
$r_{1}($ side $)$ & 0.335 & $\mp 0.003$ \\
$r_{1}($ back $)$ & 0.349 & $\mp 0.003$ \\
$r_{2}($ pole $)$ & 0.260 & $\mp 0.002$ \\
$r_{2}($ side $)$ & 0.265 & $\mp 0.002$ \\
$r_{2}$ (back) & 0.274 & $\mp 0.003$ \\
\hline
\end{tabular}

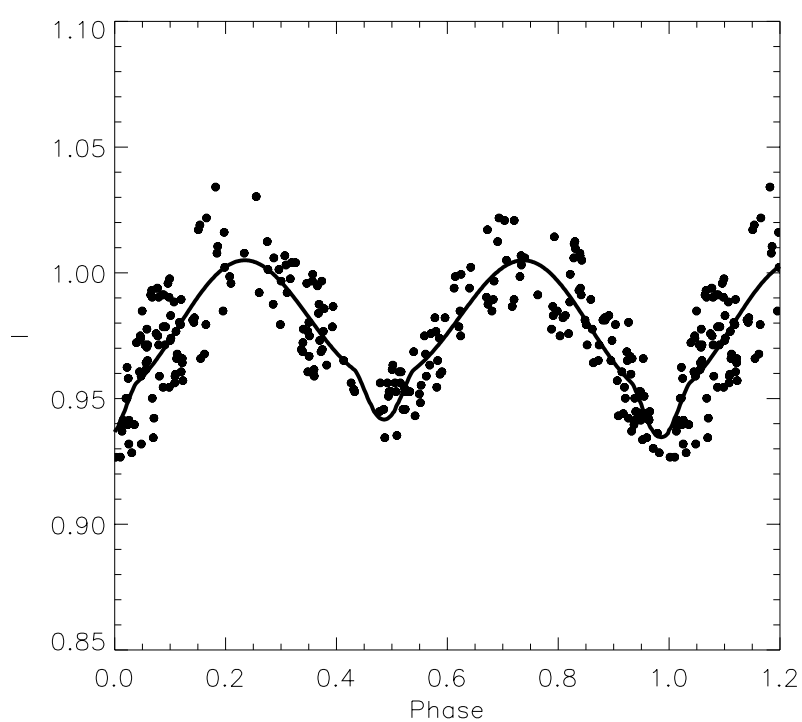

Fig. 6. A comparison of the observed (dots) and computed (solid line) $B$ light curves of V497 Cep.

Table 5. Absolute parameters for V497 Cep. Errors of the last two digits are given in parentheses.

\begin{tabular}{llll}
\hline \hline Parameter & & Primary & Secondary \\
\hline$M$ & {$\left[M_{\odot}\right]$} & $6.89(46)$ & $5.39(40)$ \\
$R$ & {$\left[R_{\odot}\right]$} & $3.69(03)$ & $2.92(03)$ \\
$T$ & {$[\mathrm{~K}]$} & $19500($ fixed $)$ & $17756(405)$ \\
$L$ & {$\left[L_{\odot}\right]$} & $1842_{-426}^{+512}$ & $725_{-185}^{+226}$ \\
\hline
\end{tabular}

of V497 Cep are little evolved main-sequence stars, with the primary being more evolved from the zero-age main sequence than the secondary as expected. The inherent remaining uncertainties of our solution does not allow us to use this comparison to an accurate determination of the age of V497 Cep. Yet, it is clear that our result does not contradict the evolutionary age of NGC 7160 cluster, $7.08 \times 10^{6} \mathrm{yr}$, derived by Harris \& Harris (2000).

\subsection{Rotational velocities and model profiles}

In Fig. 5 we compare the disentangled He I 6678 line profiles of both components, normalized to their individual continua
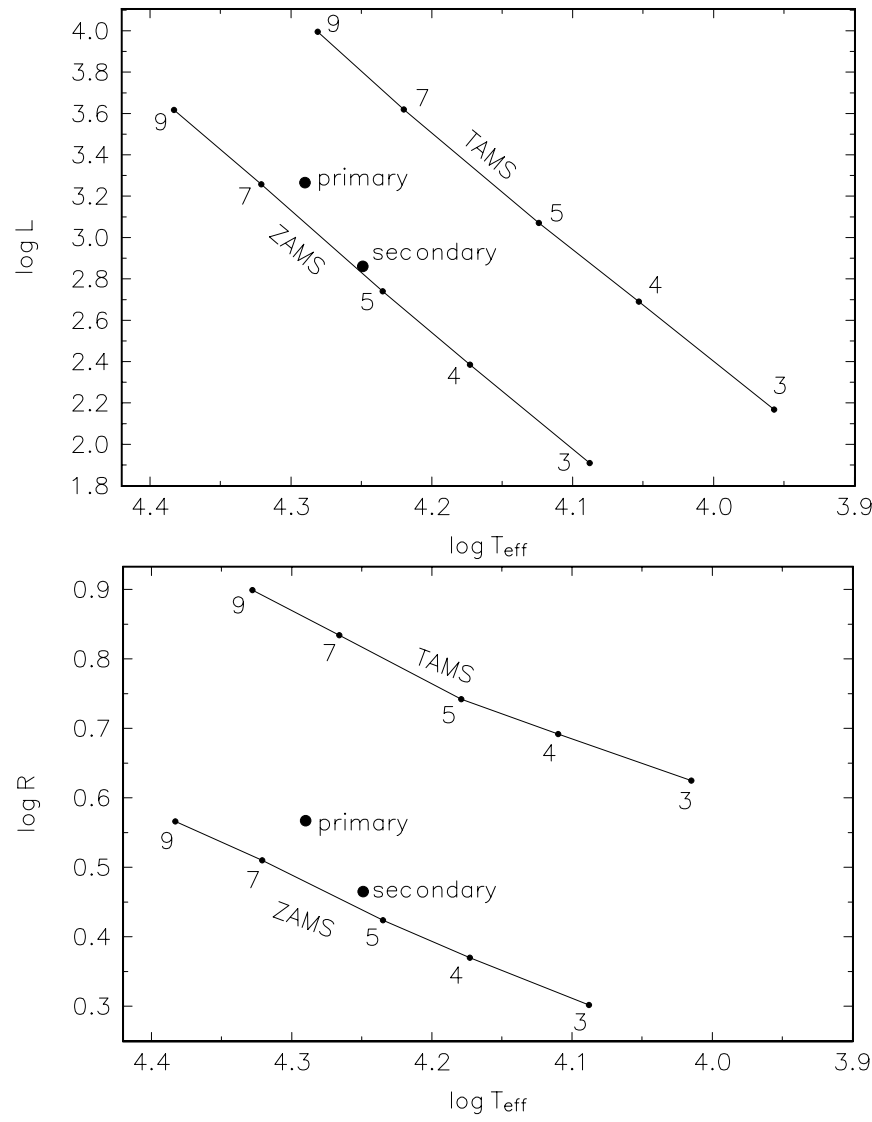

Fig. 7. The positions of the stellar components of the eclipsing, double-lined binary V497 Cep in the a) $\log L / L_{\odot}-\log T_{\text {eff }}$ and b) $\log R / R_{\odot}-\log T_{\text {eff }}$ diagrams. Masses of corresponding evolutionary models are indicated near the corresponding points with both, zeroage (ZAMS) and terminal-age (TAMS) main sequence locations.

using the $V$-band fractional luminosities of 0.651 and 0.349 , with the synthetic line profiles broadened to projected rotational velocities of 132 and $104 \mathrm{~km} \mathrm{~s}^{-1}$, corresponding to spin-orbit synchronization. Synthetic spectra for our analysis were calculated from Kurucz's (1993b) grid of solar composition LTE line-blanketed model atmospheres with the help of a computer code SYNSPEC (for its description see Hubeny et al. 1994). Oscillator strengths, wavelengths and damping parameters for all lines contributing to the resulting spectrum in the neighbourhood of the line profiles in question were taken from the list of Kurucz (1993a). The synthetic spectra resulting from SYNSPEC were rotationally broadened with the help of SPEFO program. Model atmospheres for log $g=4.0$ and effective temperatures of $20000 \mathrm{~K}$ and $18000 \mathrm{~K}$, closest to our estimated properties of the primary and secondary of V497 Cep, were used.

The line of He I 6678 is known to be prone to NLTE effects, therefore the less perfect agreement in the line core of the primary between the observed and synthetic profile is not very disturbing. Note also that we had to use a synthetic profile corresponding to somewhat higher effective temperature than what we believe is the true effective temperature of the primary. For the secondary, the agreement is nearly perfect. 
In any case, one can see that the widths of the observed and model line profiles agree perfectly for both stars. This implies that both binary components indeed rotate with angular velocities synchronized with orbital revolution as expected for such a close binary. In turn, it represents another consistency check of our orbital and light-curve solutions.

\section{Discussion}

The new light and radial velocity curves of V497 Cep were analysed by means of modern methods. It was found that the light variability of the system is mainly due to the oblateness of the components. In addition to the light variation arising from the ellipticity effect, there is a a small contribution to the light variation arising from the grazing eclipses, first detected in this study. Analyses of both light and RV curves yield the geometrical elements of the orbit as well as physical parameters of the stellar components. Combining the results of both analyses we obtained also absolute dimensions and masses of both stars. A comparison with evolutionary models clearly indicates that both stars are little evolved from the theoretical zero-age main sequence. This result is in agreement with the available estimates of the age of open cluster NGC 7160.

Using the fractional luminosities of the components from the $B$ and $V$ light-curve solutions, quoted above, and adopting the observed $V$ and $B$ magnitudes of the system at maximum light, $V=8$. 945 and $B=9.9 \mathrm{~m} .137$ and standard dereddening, which leads to $E(B-V)=0 \mathrm{~m} \cdot 39$, we find the following dereddened values for the primary and secondary:

$V_{0}=8 \cdot 14, \quad(B-V)_{0}=-0 \cdot 204$

$V_{0}=8 \cdot 81, \quad(B-V)_{0}=-0 \cdot 197$.

Adopting the BC corrections after Drilling \& Landolt (2000), -2.13 and -1.86 , one gets the distance modulus of 9.43 and 9.37 for the primary and secondary, respectively which corresponds to the distance of about $770_{-95}^{+100} \mathrm{pc}$ for the primary, and $750_{-102}^{+109} \mathrm{pc}$ for the secondary component. The mean distance to the cluster is about $760 \pm 100$ pc. Conti \& van den Heuvel (1970) derived a distance to the cluster of $830 \mathrm{pc}$, a mean $E_{B-V}=0.38$ and an age of 18 million years. However, the mean reddening and distance of the cluster are given by Lynga (1987) as $0 \mathrm{~m} 375$ and $900 \mathrm{pc}$, respectively. On the other hand, Harris \& Harris (2000) give a reddening of 0.36 and distance of $750 \mathrm{pc}$ for the open cluster NGC 7160 . Our results are in agreement with the above-mentioned results except that our determination puts NGC 7160 to a smaller distance than Lynga's estimate. The fact that V497 Cep is a confirmed member of NGC 7160 cluster underlines the astrophysical importance of V497 Cep binary. Given the fact that we were able to obtain a physically consistent solution indicates that also our new distance determination should be taken seriously, although further tests are clearly desirable. In particular, new very accurate $U B V$ light curves which would allow solutions in all three passbands, and modelling of disentangled spectra obtained over a much wider interval of wavelengths, would help to improve the accurate knowledge of stellar effective temperatures and a better estimate of deredened $U B V$ values.

Acknowledgements. PH gratefully acknowledges the warm hospitality during his 2002 visit to Department of Astronomy and Space Sciences of Ege University where some aspects of this paper were discussed. DH would like to thank the director of the Dominion Astrophysical Observatory for generous allocation of observing time. This study has been supported by Ege University Research Fund (Project No. 99/FEN/016 and 2002/FEN/002). It is also partly based on the observational data secured by $\mathrm{PH}$ and $\mathrm{PE}$ in the course of the Czech-Mexican KONTAKT/CONACyT collaborative project E130.734. In the early stages, it was carried out as a part of the research supported by the grants 205/96/0162 and 205/02/0445 of the Grant Agency of the Czech Republic. In later stages, PH was also supported via research plans J13/98: 113200004 of MŠMT, and AV 0Z1 003909 and via project K2043105 of AV ČR. Finally, we express our thanks to the referee, Dr. C. Lazaro, for his helpful, constructive criticism which helped to improve the paper.

\section{References}

Claret, A. 2000, A\&A, 363, 1081

Conti, P. S., \& van den Heuvel, E. P. J. 1970, A\&A, 9, 466

Drilling, J. S., \& Landolt, U. A. 2000, in Allen's Astrophysical Quantities, Fourth Edition, ed. A. N. Cox (Springer), 388

Hadrava, P. 1995, A\&AS, 114, 393

Hadrava, P. 1997, A\&AS, 122, 581

Hadrava, P. 1990, Contr. Astron. Obs. Skalnaté Pleso, 20, 23

Harmanec, P., Horn, J., \& Juza, K. 1994, A\&AS, 104, 121

Harmanec, P., Božić, H., Eenens, P., \& Žižňovský, J. 1999, A\&A, 346, 87

Harris, H. C., \& Harris, W. E. 2000, in Allen's Astrophysical Quantities, Fourth Edition, ed. A. N. Cox (Springer), 545

Hayford, P. 1932, Lick Obs. Bull. 16, 53

Hill, G. 1967a, ApJS, 14, 263

Hill, G. 1967b, ApJS, 14, 301

Hill, G., Hilditch, R. W., \& Pfannenschmidt E. L. 1976, Publ. Dom. Astrophys. Obs. 15, 1

Holmgren D. E., \& Tarasov, A. E. 2000, J. Royal Astr. Soc. Canada, 94, 248

Horn, J., Kubát, J., Harmanec, P., et al. 1996, A\&A, 309, 521

Hubeny, I., Lanz, T., \& Jeffery, C. S. 1994, in Newsletter on Analysis of Astronomical Spectra No. 20, ed. by C. S. Jeffery (St. Andrews University), 30

Kazarovets, E. V., Samus, N. N., \& Durlevich, O. V. 2000, Inf. Bull. Var. Stars, No. 4870

Kurucz, R. L. 1993a, Atomic Data for Opacity Calculations, Kurucz CD-ROM No. 1

Kurucz, R. L. 1993b, Solar Abundance Model Atmospheres, Kurucz CD-ROM No. 19

Lynga, G. 1987, Catalogue of Open Cluster Data, 5th ed. (a computerbased catalogue distibuted by NASA Data Center)

Rucinski, S. M. 1969, Acta Astron., 19, 245

Schaller, G., Schaerer, D., Meynet, G., \& Maeder, A. 1992, A\&AS, 96, 269

von Zeipel, H. 1924, MNRAS, 84, 665

Wilson, R. E., \& Devinney, E. J. 1971, ApJ, 166, 605

Wilson, R. E. 1994, PASP, 106, 921 